\title{
PROPRIEDADES DE DISPERSÕES DE SURFACTANTES PARA A SÍNTESE DE SÍLICAS HÍBRIDAS
}

\author{
L. B. OLIVEIRA ${ }^{1}$, L. L. SILVA ${ }^{1}$, A. F. P. CAMPOS ${ }^{2}$ e D. CARDOSO ${ }^{1}$ \\ ${ }^{1}$ Universidade Federal de São Carlos, Departamento de Engenharia Química \\ ${ }^{2}$ Centro Universitário do Planalto de Araxá, UNIARAXA, Brasil. \\ E-mail para contato: dilson@ufscar.br
}

\begin{abstract}
RESUMO - Dentre as várias peneiras moleculares pertencentes à família M41S e de grande interesse como catalisadores heterogêneos mesoporosos, foi comprovado que à sílica MCM-41, quando não submetida a calcinação, apresenta forte caráter básico devido ao grupo silóxi $\left(\equiv \mathrm{SiO}^{-}\right)$presente na parede dos seus mesoporos. Na síntese dessa sílica é usualmente utilizado o brometo de cetiltrimetilamônio (CTABr), entretanto, com a modificação do surfactante utilizado na síntese, estudos mostram influências na sílica híbrida obtida, já que o surfactante atua como agente direcionador na formação de sua estrutura. Nesse contexto, este trabalho apresenta as propriedades das dispersões de surfactantes alternativos e interessantes a síntese da MCM-41. Determinou-se a concentração micelar crítica $(\mathrm{CMC})$ a $25^{\circ} \mathrm{C}$, ainda não registrada na literatura, dos brometos de: tetradeciltripropilamônio $(\mathrm{C} 14 \mathrm{Pr} 3 \mathrm{Br})$, cetiltripropilamônio $(\mathrm{C} 16 \mathrm{Pr} 3 \mathrm{Br})$, octadeciltripropilamônio $(\mathrm{C} 18 \mathrm{Pr} 3 \mathrm{Br})$, eicosanotripropilamônio $(\mathrm{C} 20 \mathrm{Pr} 3 \mathrm{Br})$ e docosanotripropilamônio $(\mathrm{C} 22 \mathrm{Pr} 3 \mathrm{Br})$. Além disso, para o $\mathrm{C} 16 \mathrm{Pr} 3 \mathrm{Br}$ também foi obtido a CMC a 40 e $50^{\circ} \mathrm{C}$, a fim de estudar a influência da temperatura na formação das micelas.
\end{abstract}

\section{INTRODUÇÃO}

Industrialmente a produção de biodiesel é viabilizada pela catálise homogênea, utilizando, preferencialmente, o metóxido de sódio. Entretanto este é miscível com o produto da reação, sendo necessário processos de separação do produto com alto custo (Lotero et al., 2005). Estudos como o de Georgogianni et al. (2009) mostraram que catalisadores heterogêneos também promovem a reação de transesterificação, mas com a vantagem de utilizar condições mais brandas. Além disso, na catálise heterogênea, há a diminuição dos custos de produção e da quantidade de resíduos gerados, visto que esta rota é menos corrosiva; os produtos da reação se apresentam em diferentes fases, simplificando a separação do produto sendo possível a recuperação e a reutilização do catalisador (Liu et al., 2008; Antune et al., 2008).

Neste contexto a sílica híbrida CTA-MCM-41 se destaca. Kubota et al. (2004) e Martins et al. (2006) verificaram que a sua atividade catalítica é básica e deve-se a presença dos grupos silóxi $\left(\equiv \mathrm{SiO}^{-}\right)$na sua estrutura. Os pesquisadores Cardoso et al. (2014) comprovaram a possibilidade de sintetizar sílicas híbridas do tipo MCM-41 e MCM-48 substituindo o usual $\mathrm{CTABr}$ por outros surfactantes catiônicos semelhantes, também do tipo amônio quaternário. 
Ainda se verificou (Campos et al., 2016) que a utilização de surfactantes catiônicos com diferentes tamanhos de cabeça e cauda têm influência nas características das sílicas sintetizadas, dentre elas: diâmetro de poros, organização da estrutura, e até mesmo na atividade catalítica, quando aplicadas na reação modelo de transesterificação.

Os surfactantes possuem em suas moléculas tanto uma região polar, quanto uma região apolar. Este fato lhes conferem um caráter anfifílico, ou seja, afinidade tanto por moléculas polares, bem como com apolares. No caso dos surfactantes do tipo amônio quaternário - isto é, aminas com o nitrogênio quaternizado - é amplamente difundido na literatura a seguinte nomenclatura: cauda para a região apolar e cabeça para a região apolar da molécula. $\mathrm{Na}$ grande maioria dos casos a interface dessas duas regiões coincide com o nitrogênio quaternário da estrutura.

$\mathrm{Na}$ presença de solventes e em pequenas quantidades, os surfactantes catiônicos se encontram independentemente solubilizados, formando uma solução eletrolítica. Entretanto, a partir de uma concentração característica formam-se, espontaneamente, agregados de menor energia denominados micelas, originando, então, uma dispersão micelar. Essa concentração mínima característica, é referida na literatura como concentração micelar crítica ou simplesmente CMC (Oseliero Filho, 2013).

Conforme descrito por Attwood e Florence (1983), a formação de micelas deve-se a característica anfifílica dos surfactantes. São várias as abordagens utilizadas para explicar esse efeito, a mais recorrente é o efeito repulsivo entre o surfactante e o solvente. Em um solvente polar, como por exemplo a água, a micela se forma pelo efeito hidrofóbico entre o solvente e a cauda do surfactante, de modo que a cauda se orienta para o interior da micela, enquanto a cabeça compõe a interface micela-solvente. No caso de um solvente apolar, como o óleo, o surfactante sofre o efeito lipofóbico e tem orientação inversa à citada anteriormente, neste último caso costuma-se denominar estes agregados como "micelas reversas".

Sendo assim, este trabalho visa estabelecer a concentração micelar crítica (CMC), em água, de diferentes surfactantes catiônicos, interessantes à síntese de sílicas híbridas. Os surfactantes catiônicos utilizados neste trabalho foram sintetizados pelo grupo de pesquisa LabCat (DEQ/UFSCar) utilizando a metodologia de Campos (2015), são estes os brometos de: tetradeciltripropilamônio (C14Pr3Br), cetiltripropilamônio (C16Pr3Br), octadeciltripropilamônio $\quad(\mathrm{C} 18 \mathrm{Pr} 3 \mathrm{Br}), \quad$ eicosanotripropilamônio $\quad(\mathrm{C} 20 \mathrm{Pr} 3 \mathrm{Br}) \quad \mathrm{e}$ docosanotripropilamônio (C22Pr3Br); além destes também utilizou-se o cetiltrimetilamônio (CTABr), obtido comercialmente (Aldrich, pureza > 98\%). Determinou-se a CMC para todos os surfactantes a $25^{\circ} \mathrm{C}$, além disso, para o C16Pr3Br determinou-se também a CMC em 40 e $50^{\circ} \mathrm{C}$, a fim de avaliar a influência da temperatura na formação das micelas.

\section{METODOLOGIA}

A detergência, pressão osmótica, condutividade molar, condutividade elétrica e tensão superficial são algumas das propriedades físico-químicas que, em função da concentração, fornecem a CMC (Oseliero Filho, 2013). Neste trabalho utilizou-se a condutividade elétrica para determinar a CMC. Esta é largamente utilizada devido a sua acurácia e facilidade de execução. 
Para o experimento foi preparada a solução concentrada e, em seguida, realizada as medidas de condutividade para cada um dos surfactantes. O sistema é constituído por um reator de vidro encamisado, que comporta o analito, acoplado ao banho termostático, a fim de manter a temperatura constante ao longo do experimento $\left( \pm 0,1^{\circ} \mathrm{C}\right)$. O reator é fixado, com uma garra, sobre o agitador magnético, usado para assegurar a homogeneidade do analito. Também com uma garra, acopla-se a bureta para gotejar a dispersão de surfactante no reator, conforme a concentração previamente estipulada.

A condutividade elétrica foi medida a cada variação de concentração da dispersão contida no reator, utilizando um condutivímetro portátil - Quimis Q795P - calibrado com solução padrão comercial (Quimis $74 \mu \mathrm{S} . \mathrm{cm}^{-1}$ ) antes de cada análise. As amostras foram preparadas a temperatura ambiente $\left(25 \pm 5^{\circ} \mathrm{C}\right)$ em balão volumétrico. Todos os experimentos foram realizados em triplicata.

Inicialmente são colocados $20 \mathrm{~mL}$ de água destilada no reator - volume necessário para que os eletrodos da sonda fiquem totalmente submersos e seja possível fazer a leitura da condutividade. Goteja-se, então, um dado volume do surfactante no reator e, após o sistema entrar em equilíbrio, é medido o valor da condutividade. Esse procedimento é repetido sistematicamente, até se obter pontos suficientes para a determinação da CMC.

Os dados foram plotados em gráficos de concentração molar por condutividade elétrica e, posteriormente, ajustados pela equação 1, cujo parâmetro $x_{0}$ equivale a CMC conforme o método de apresentado por Carpena et al. (2002).

$$
F(x)=F_{0}+A_{1} x+\Delta x\left(A_{1}-A_{2}\right) \ln \left(\frac{1+e^{\frac{x-x_{0}}{\Delta x}}}{1+e^{\frac{-x_{0}}{\Delta x}}}\right)
$$

\section{RESULTADOS E DISCUSSÃO}

A Figura 1 apresenta os gráficos da condutividade elétrica pela concentração molar de surfactantes que possuem a mesma cabeça e diferentes tamanhos de cauda $\left(\mathrm{C}_{n} \mathrm{Pr}_{3} \mathrm{Br}\right.$ com $\mathrm{n}=$ $14,16,18,20$ e 22 carbonos), à $25^{\circ} \mathrm{C}$ e também para o CTABr. Os dados foram ajustados utilizando a equação 1, segundo o método de Carpena (2002).

Na Figura 1, para todos os surfactantes, nota-se que conforme há o aumento progressivo da concentração de surfactante, ocorre o aumento linear da condutividade. Isto deve-se ao aumento da concentração de íons, espécies químicas responsáveis pelo transporte de elétrons na solução. Quando há a formação de agregados micelares a variação da condutividade elétrica também é linear, entretanto com uma taxa de variação linear (representada graficamente pela tangente) inferior a obtida sem a formação de micelas. 
Figura 1 - Gráfico da concentração molar por condutividade, à $25^{\circ} \mathrm{C}$, dos surfactantes: a) $\mathrm{C}_{14} \mathrm{Pr}_{3} \mathrm{Br}$; b) $\mathrm{C}_{16} \mathrm{Pr}_{3} \mathrm{Br}$; c) $\mathrm{C}_{18} \mathrm{Pr}_{3} \mathrm{Br}$; d) $\mathrm{C}_{20} \mathrm{Pr}_{3} \mathrm{Br}$; e) $\mathrm{C}_{22} \mathrm{Pr}_{3} \mathrm{Br}$; f) CTABr.

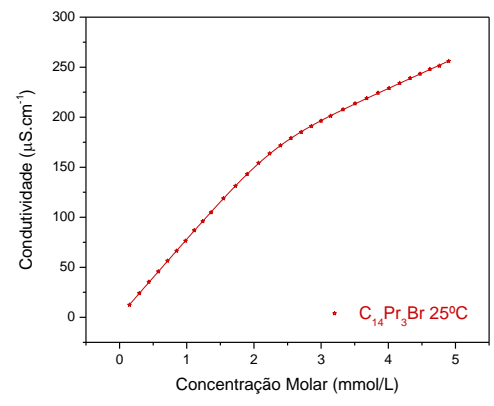

a)

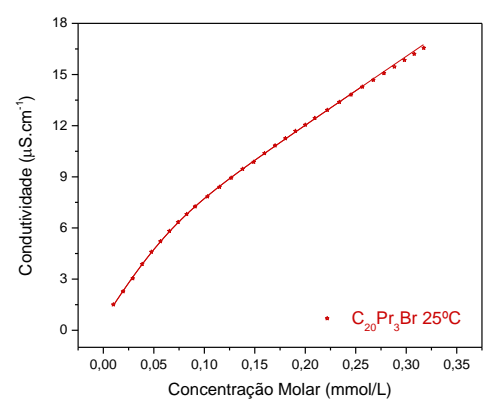

d)

e)

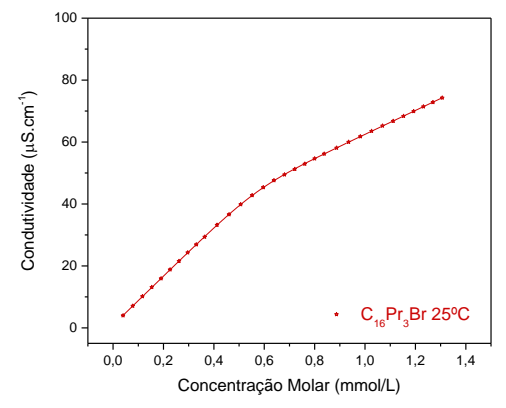

b)

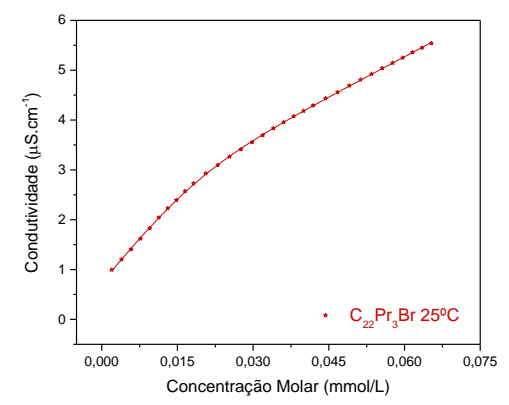

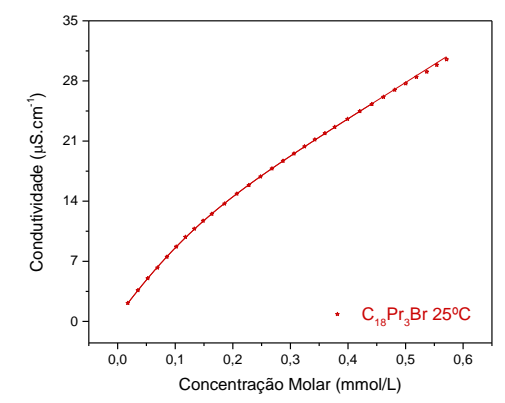

c)

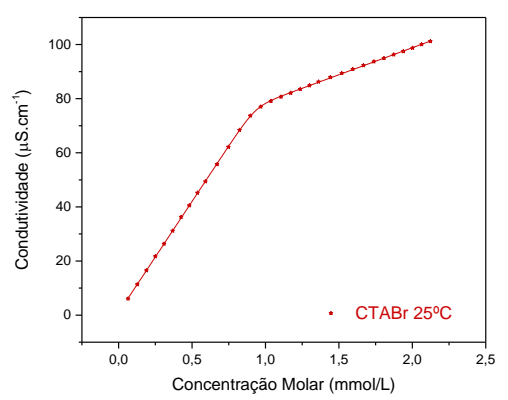

f)

Isto ocorre devido a menor mobilidade dos agregados micelares em comparação as moléculas independentemente dissociadas e também aos contraíons aniônicos associados as micelas que, ao contribuírem na estabilização elétrica do agregado, neutralizam parte das cargas positivas dos surfactantes que compõem a micela resultando, portanto, em um incremento na condutividade inferior à observada em baixas concentrações, sem a presença de micelas (Attwood e Florence 1983). Este fato explica a descontinuidade do regime linear alteração da tangente - observada no processo de micelização.

A concentração micelar crítica obtida para cada surfactante está indicada na Tabela 1.

Tabela 1 - Concentração Micelar Crítica de Surfactantes Triprolilamônio à $25^{\circ} \mathrm{C}$.

\begin{tabular}{|c|c|c|c|c|c|c|}
\hline Surfactante & $\mathrm{C}_{14} \mathrm{Pr}_{3} \mathrm{Br}$ & $\mathrm{C}_{16} \mathrm{Pr}_{3} \mathrm{Br}$ & $\mathrm{C}_{18} \mathrm{Pr}_{3} \mathrm{Br}$ & $\mathrm{C}_{20} \operatorname{Pr}_{3} \mathrm{Br}$ & $\mathrm{C}_{22} \mathrm{Pr}_{3} \mathrm{Br}$ & $\mathrm{CTABr}$ \\
\hline $\mathbf{C M C}(\mathbf{m m o l} / \mathbf{L})$ & 2,26 & 0,57 & 0,07 & 0,05 & 0,02 & 0,92 \\
\hline
\end{tabular}

Pela Tabela 1 verifica-se que, mantendo a cabeça tripropil fixa $(\operatorname{Pr} 3)$, a CMC diminui com o aumento da cauda apolar do surfactante. Como discutido anteriormente, a formação da micela deve-se principalmente ao efeito hidrofóbico (repulsivo) que a água exerce na parte apolar das moléculas dos surfactantes, sendo que a auto-organização do sistema em micelas leva a uma menor área de contato entre a água e a cauda apolar hidrofóbica e, consequentemente, para um estado com menor Energia de Gibbs, por isso ocorre este 
processo espontaneamente. Sendo assim, um surfactante com maior cauda possui maior área de repulsão, de modo que há a formação de micelas em menores concentrações, ou seja, menor CMC, conforme observado experimentalmente.

Ainda pela Tabela 1, temos o CTABr e o $\mathrm{C}_{16} \mathrm{Pr}_{3} \mathrm{Br}$, ambos os surfactantes com cauda cetil, diferindo apenas na cabeça (o primeiro trimetil e o segundo tripropil). Os resultados indicam que o aumento da cabeça de um surfactante implica em uma menor CMC.

Para avaliar a influência da temperatura na formação de micelas, foi determinada a $\mathrm{CMC}$ do $\mathrm{C}_{16} \mathrm{Pr}_{3} \mathrm{Br}$ a diferentes temperaturas. A Figura 2 apresenta os gráficos da condutividade elétrica pela concentração molar do $\mathrm{C}_{16} \mathrm{Pr}_{3} \mathrm{Br}$ à 25,40 e $50{ }^{\circ} \mathrm{C}$, enquanto que a Tabela 2 apresenta as CMC's obtidas.

Figura 2 - Gráfico da concentração molar por condutividade do $\mathrm{C}_{16} \operatorname{Pr}_{3} \mathrm{Br}$ à:

a) $25^{\circ} \mathrm{C}$; b) $40{ }^{\circ} \mathrm{C}$; c) $50{ }^{\circ} \mathrm{C}$.

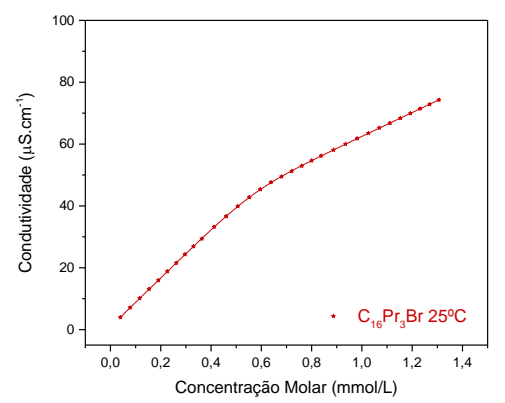

a)

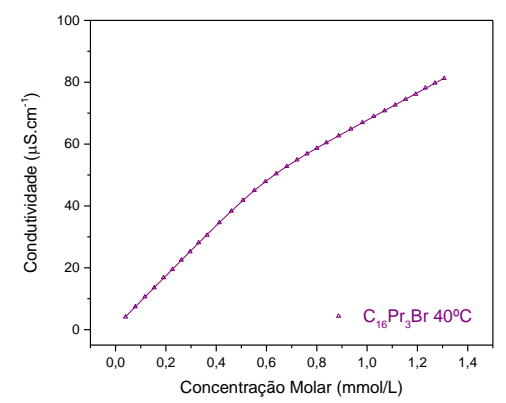

b)

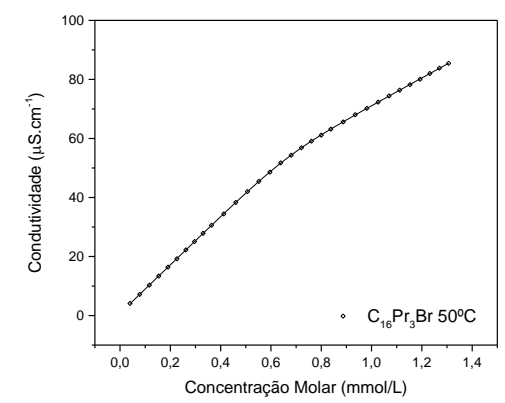

c)

Tabela 2 - Concentração Micelar Crítica do $\mathrm{C}_{16} \mathrm{Pr}_{3} \mathrm{Br}$ em diferentes temperaturas.

\begin{tabular}{|c|c|c|c|}
\hline Temperatura $\left({ }^{\circ} \mathbf{C}\right)$ & 25 & 40 & 50 \\
\hline $\mathbf{C M C}(\mathbf{m m o l} / \mathbf{L})$ & 2,26 & 0,57 & 0,07 \\
\hline
\end{tabular}

A temperatura é um indicativo do grau de agitação das moléculas, portanto o seu aumento reflete no aumento de entropia do sistema. Como as micelas são agregados organizados, para a sua formação em maiores temperaturas é necessária uma maior concentração de surfactante, até que o aumento da repulsão hidrofóbica pela quantidade de moléculas, compense o ganho de entropia do sistema pelo aumento da temperatura.

\section{CONSIDERAÇÕES FINAIS}

Com o aumento da cauda do surfactante, a micela é formada mais facilmente, ou seja, houve a diminuição da CMC. Pois a maior área apolar de um surfactante intensifica o efeito hidrofóbico. $\mathrm{O}$ aumento da temperatura dificulta a formação de micelas, influenciando no aumento do valor da CMC, isto deve-se ao maior grau de agitação das moléculas que dificultam a estabilização dos agregados micelares do surfactante.

A CMC obtida experimentalmente para o $\mathrm{CTABr}$ foi idêntica à informada pelo fabricante (Aldrich), indicando que o método utilizado foi satisfatório. 
A determinação das propriedades de sistemas micelares é o início para a compreensão da influência do surfactante na síntese de sílicas híbridas com estrutura MCM-41. Devido a função do surfactante como agente direcionador de poros à formação da estrutura da sílica, pelo mecanismo cooperativo. $\mathrm{O}$ estudo da $\mathrm{CMC}$ desses surfactantes auxilia na identificação de condições ideais de síntese (como temperatura e solvente).

\section{REFERÊNCIAS}

ANTUNE, W.M.; VELOSO, C.O.; HENRIQUES, C.A. Transesterification of soybean oil with methanol catalyzed by basic solids. Catal. Today, v. 133-135, p. 548-554, 2008.

ATTWOOD, D.; FLORENCE, A. T. Surfactant systems: Their chemistry, pharmacy and biology. London: Chapman \& Hall, 1983

CAMPOS, A. F. P. Síntese e propriedades de sílicas híbridas contendo surfactantes catiônicos, Tese, PPGEQ, UFSCar, São Carlos, 206 p., 2015.

CAMPOS, A. F. P.; FERREIRA, A. R. O.; CARDOSO, D. Síntese e propriedades de sílicas híbridas contendo surfactantes cetiltrialquilamônio. Quim. Nova, v. 39, p. 279-285, 2016.

CARDOSO, D.; SILVA, L. L.; CRUZ, I. H.; ARAÚJO, J., Patente INPI: BR 102014002430 $-1,2014$

CARPENA P., AGUIAR J., BERNAOLA-GALVÁN P., RUIZ C. C. Problems Associated with the Treatment of Conductivity-Concentration Data in Surfactant Solutions: Simulations and Experiments. Langmuir, v. 18, p. 6054, 2002.

GEORGOGIANNI, K. G.; KATSOULIDIS, A. K.; POMONIS, P. J.; MANOS, G.; KONTOMINAS, M. G. Transesterification of rapeseed oil for the production of biodiesel using homogeneous and heterogeneous catalysis. Fuel Processing Technology, v. 90, p. 1016-1022, 2009.

KUBOTA, Y.; NISHIZAKI, Y.; IKEYA, H.; SAEKI, M.; HIDA, T.; KAWAZU; S.; Yoshida, M.; FUJII, H.; SUGI, Y.; Organic-silicate hybrid catalysts based on various defined structures for Knoevenagel condensation. Microporous and Mesoporous Mater., v. 70, p. 135-149, 2004.

LIU, R.; WANG, X.; ZHAO, X., FENG, P. Sulfonated ordered mesoporous carbon for catalytic preparation of biodiesel. Carbon, v. 46, p.1664-1669, 2008.

LOTERO, E.; LIU, Y.; LOPEZ, D. E.; SUWANNAKARN, K.; BRUCE, D. A.; GOODWIN JR, J. G. Synthesis of Biodiesel via Acid Catalysis. Ind. Eng. Chem. Res., v. 44, p.53535363, 2005.

MARTINS, L.; BONAGAMBA, T. J.; AZEVEDO, E. R.; BARGIELA, P.; CARDOSO, D. Surfactant containing Si-MCM-41: An efficient basic catalyst for the Knoevenagel condensation. Appl. Catal. A, v. 312, p. 77-85, 2006.

OSELIERO FILHO, P. L.; Estudo Estrutural e Termodinâmico de Sistemas AutoOrganizados: Micelas em Solução. Dissertação. Departamento de Física Experimental, USP, São Paulo, 112 p., 2013. 